\title{
Carbon Nanotubes in Cementitious Composites: Dispersion, Implementation, and Influence on Mechanical Characteristics
}

\author{
Tomas Jarolim, Martin Labaj, Rudolf Hela, and Kamila Michnova \\ Faculty of Civil Engineering, Institute of Technology of Building Materials and Components, Brno University of Technology, \\ Veveri 331/95, 60200 Brno, Czech Republic
}

Correspondence should be addressed to Tomas Jarolim; jarolim.t@fce.vutbr.cz

Received 18 February 2016; Revised 23 June 2016; Accepted 14 July 2016

Academic Editor: Gonzalo Martínez-Barrera

Copyright (C) 2016 Tomas Jarolim et al. This is an open access article distributed under the Creative Commons Attribution License, which permits unrestricted use, distribution, and reproduction in any medium, provided the original work is properly cited.

For effective utilization of nanoparticles in cementitious composite materials, their perfect dispersion is necessary. Appropriate quantity of ultrasonic energy (US energy), as well as usage of proper and compatible surfactant, is a prerequisite for carbon nanotubes' (CNT) suitable deagglomeration. US energy was supplied by Bandelin Sonopuls HD 3200 ultrasonic homogenizer. Influence of US energy's amount on CNT's dispersion in aqueous medium was monitored in two steps: at first roughly by an optical microscope and in the next step precisely by the measurement of absorbance via UV/Vis spectrophotometry. After the appropriate amount of US energy was found, cement mortars with CNT were prepared and tested. Their physical and mechanical properties were examined and compared to the reference specimens.

\section{Introduction}

Nowadays, there are not many new raw materials which could be incorporated into cementitious materials (e.g., concrete) to increase their strength characteristics and durability and decrease their carbon footprint [1-4]. Due to technological progress in a wide spectrum of scientific fields (chemistry, electrical engineering, IT, medicine, optics, etc.), we can now produce nanoparticles using the bottom-up approach. This method means a breakthrough in material engineering because it makes materials' production with accurately defined parameters (and rather interesting ones) possible. Therefore, nanotechnology is one of the fastest developing scientific fields. CNT can be used as a matrix of reinforcement in many industrial sectors like metallurgy $[5,6]$, polymer industry $[7,8]$, and, of course, concrete industry $[9,10]$. There are other nanomaterials with potentially interesting effects on cementitious composites, for example, nanosilica. Many experiments with nanosilica were performed. Their results confirm that implementation of nanosilica to the cement matrix has positive impact to the cement composites' mechanical characteristics [11, 12]. However, this paper is focused mainly on CNT's implementation into the cementitious composites as a scattered reinforcement of cement matrix. In the beginning, problems regarding dispersion's stability and potential toxicity as well as financial availability need to be addressed $[13,14]$.

\section{Introduction to the Issue of Carbon Nanotubes}

2.1. Carbon Nanotubes (CNT). Carbon nanotubes can be characterized as graphene sheets rolled into a cylindrical tube with a length of 1 to $100 \mu \mathrm{m}$. These structures were initially identified in 1991 by Japanese scientist Iijima [15]. Carbon nanotubes can be produced using several methods, for example, electric arc discharge, laser ablation, and chemical vapor deposition. The last mentioned one is currently the most used method. Carbon nanotubes have very good physicalmechanical properties $[16,17]$. According to the number of internal layers, CNT are divided into a single-walled carbon nanotubes (SWCNT) and multiwalled carbon nanotubes (MWCNT). SWCNT typically contain one graphene sheet and have an outer diameter of approximately 5-20 nm. MWCNT have an inner diameter of approximately $1.5-15 \mathrm{~nm}$ and an outer diameter of up to $100 \mathrm{~nm}$. CNT have exceptional properties which give them potential to become as important 
for the development of industry as the discovery of the steam engine. Their typical properties are elastic modulus of approximately $1 \mathrm{TPa}$, tensile strength in a range of 11-63 GPa, volumetric weight of about $1300 \mathrm{~kg} \cdot \mathrm{m}^{-3}$, and surface area of $70-400 \mathrm{~m}^{2} \cdot \mathrm{g}^{-1}[18,19]$.

2.2. Dispersing Carbon Nanotubes. CNT tends to form bundles; thus, for perfect implementation to the cement matrix, the flawless dispersion and uniform distribution are essential factors [16]. Currently, there are two main approaches for dispersing nanotubes: mechanical methods and chemical methods that modify the surface energy of solids. Mechanical methods include high shear mixing and cavitation, namely, either hydrodynamic cavitation (i.e., cavitation caused by high speed liquid flow) or acoustic/ultrasonic cavitation induced by passing of high energy acoustic waves through liquid medium. Literature mentions the possibility of combining methods for dispersing CNT, for example, ultrasonic method and suitable surfactant. Surface-active substances are capable of preventing carbon nanotubes' reagglomeration after the ultrasonication treatment and make their disentanglement easier as well [20-22]. It is necessary to ensure that the added surfactant does not impact the setting and hardening of concrete or cement mortar. CNT's optimal dispersion is one of the key factors for preparation of enhanced cement based composite materials. CNT possess an enormous surface energy and an extremely high aspect ratio and are attracted to each other with van der Waals forces. These facts result in their strong tendency to form agglomerates/bundles. Bundles reduce the material's overall strength, because they create weak spots in the matrix, in which the initial cracks are developing after loading [23].

A relatively new phenomenon is CNT with surfactants incorporated in their structure. When CNT are dispersed in aqueous medium via ultrasonication, it is important to make sure that the supplied acoustic energy is not exceeded, because otherwise (depending on the CNT's concentration) the structure of graphene sheets could be damaged or even broken. CNT's deformation reduces expected positive impact on physicomechanical properties of specimens enhanced with CNT $[25,26]$.

\section{Experimental Part}

The main goal of the experiment was to create stable dispersion of CNT, water, and appropriate surfactant and then use it to enhance the cement mortar's properties. Research's structure was designed with consideration of previous experimental work, as well as scientific papers [19-23, 25-27]. The level of CNT's deagglomeration in dispersion was measured via the optical microscope (Jenoptik ProgRes CF) and also (mainly) UV/Vis Spectrophotometer (LAMBDA 1050 UV/Vis/NIR Spectrophotometer made by PerkinElmer). Once the optimal amount of ultrasonic energy was determined, the next step was to incorporate said dispersions to cement mortars and observe the CNT's influence on their flexural and compressive strengths. Mixture of water, CNT, and surfactant was initially homogenized by a magnetic stirrer Variomag Monotherm. Subsequently, the suspension was treated by ultrasonic
TABLE 1: Properties of carbon nanotubes.

\begin{tabular}{lc}
\hline Internal diameter & Approximately $5-12 \mathrm{~nm}$ \\
Outer diameter & Approximately $30-50 \mathrm{~nm}$ \\
Length & $10-20 \mu \mathrm{m}$ \\
Bulk density & $0.22 \mathrm{~g} \cdot \mathrm{cm}^{-3}$ \\
True density & $2.1 \mathrm{~g} \cdot \mathrm{cm}^{-3}$ \\
\hline
\end{tabular}

TABLE 2: OPC's chemical composition [17].

\begin{tabular}{lc}
\hline $\mathrm{CaO}(\%)$ & 65.000 \\
$\mathrm{SiO}_{2}(\%)$ & 19.000 \\
$\mathrm{Al}_{2} \mathrm{O}_{3}(\%)$ & 4.000 \\
$\mathrm{Fe}_{2} \mathrm{O}_{3}(\%)$ & 3.000 \\
$\mathrm{MgO}(\%)$ & 1.000 \\
$\mathrm{SO}_{3}(\%)$ & 3.000 \\
$\mathrm{~S}^{-I I}(\%)$ & 0.040 \\
$\mathrm{Cl}^{-}(\%)$ & 0.051 \\
$\mathrm{~K}_{2} \mathrm{O}(\%)$ & 0.750 \\
$\mathrm{Na}_{2} \mathrm{O}(\%)$ & 0.150 \\
$\mathrm{MgO}(\%)$ & 1.400 \\
Loss on ignition (\%) & 3.100 \\
Content of insoluble residue (\%) & 0.700 \\
\hline
\end{tabular}

TABLE 3: Clinker's composition [17].

\begin{tabular}{lc}
\hline $\mathrm{C}_{3} \mathrm{~S}(\%)$ & 67 \\
$\mathrm{C}_{2} \mathrm{~S}(\%)$ & 11 \\
$\mathrm{C}_{3} \mathrm{~A}(\%)$ & 7 \\
$\mathrm{C}_{4} \mathrm{AF}(\%)$ & 11 \\
\hline
\end{tabular}

homogenizer Badelin Sonopuls HD 3200, which should ensure CNT's flawless disentanglement and distribution. $\mathrm{UV} / \mathrm{Vis}$ spectroscopy's principle is the measurement of the absorption of electromagnetic radiation of wavelength from about 200 to $800 \mathrm{~nm}$. Measured variable is called absorbancenegative transmittance's logarithm (the ratio of luminous flux passing through the environment and the luminous flux incident on the environment). At zero absorption, the absorbance is zero and vice versa [28].

3.1. Materials. The carbon nanotubes used were supplied by Chinese producer Yurui (Shanghai) Chemical Co., Ltd. These were MWCNT with a purity of more than 95\% and were produced by chemical vapor deposition method. For other properties, see Table 1. All information was taken from the supplier's product data sheet.

For CNT's stabilization, naphthalene based superplasticizer was used. As a binder, Ordinary Portland Cement (OPC) class CEM I $42.5 \mathrm{R}$ produced by the Mokra cement plant (HeidelbergCement, Czech Republic) was used. Its composition can be seen in Tables 2 and 3 [29].

CEN (from French Comité Européen de Normalisation) standardized quartz sand (with accordance to EN 196-1 [30]) was used; for its particle distribution, see Table 4 [24]. 
TABLE 4: CEN standardized sand's particle size distribution [24].

\begin{tabular}{lc}
\hline Square mesh Size $(\mathrm{mm})$ & Cumulative $(\%)$ retained \\
\hline 0.08 & $99 \pm 1$ \\
0.16 & $87 \pm 5$ \\
0.50 & $67 \pm 5$ \\
1.00 & $33 \pm 5$ \\
1.60 & $7 \pm 5$ \\
2.00 & 0 \\
\hline
\end{tabular}

TABle 5: Composition of CNT dispersions.

\begin{tabular}{lc}
\hline Component & Dosage $(\mathrm{g})$ \\
\hline Carbon nanotubes & 0.0225 \\
Water & 99.0600 \\
Surfactant (naphthalene based superplasticizer) & 0.9000 \\
\hline
\end{tabular}

TABLE 6: Material composition of cement mortars with CNT.

\begin{tabular}{lc}
\hline Component & Dosage \\
\hline CNT dispersion (distilled water, & 225 \\
MWCNT, surfactant) (g) & 450 \\
OPC 42.5 R, Mokra (g) & 1350 \\
Normalized sand CEN (g)
\end{tabular}

3.2. Methods. The suspension of carbon nanotubes was prepared by mixing all the components (CNT, distilled water and superplasticizer; see in Table 5) while being stirred with a magnetic mixer $(600 \mathrm{rpm})$. The mixture was homogenized for 5 minutes. After 5 minutes of homogenization (and probably partial dispersion), the mixture was transferred to a reaction rosette vessel and ultrasonicated for the total amount of $2000 \mathrm{~J} / \mathrm{mL}$ of acoustic energy. After each $200 \mathrm{~J} / \mathrm{mL}$, a small amount of dispersion was taken as a sample for UV/Vis spectroscopy measurement. Also a drop of dispersion was observed under the optical microscope for bundles presence (or their nonpresence). Those two methods determined the optimal acoustic energy for samples' preparation.

It is important to mention that the sonicated dispersion had to be diluted and the dilution itself is one of the most critical steps because of rapid $\mathrm{pH}$ leaps that can lead to CNT's reagglomeration. CNT's dispersion had to be diluted to the required volume of $225 \mathrm{~mL}$ for cement mortar samples preparation [19]. Around $\mathrm{pH}$ of 7, the shift in particles' zeta potential occurs. This phenomenon leads to dispersion's instability and subsequently results in CNT's reagglomeration [31].

Cement mortar specimens with dimensions of $40 \times 40 \times$ $160 \mathrm{~mm}$ were casted according to EN 196-1. Cement mortars' material composition is referred to in Table 6 [30]. After 24 hours, the specimens were demolded and placed in a curing environment $\left(20^{\circ} \mathrm{C}, 100 \%\right.$ relative humidity).

The specimens' flexural and compressive strengths were tested after 7 and 28 days of curing according to EN 196-1 [30]. Results are discussed below. A small fragment of mortar was taken and prepared for SEM microscopy observation to evaluate the possible presence CNT bundles in the matrix (as can

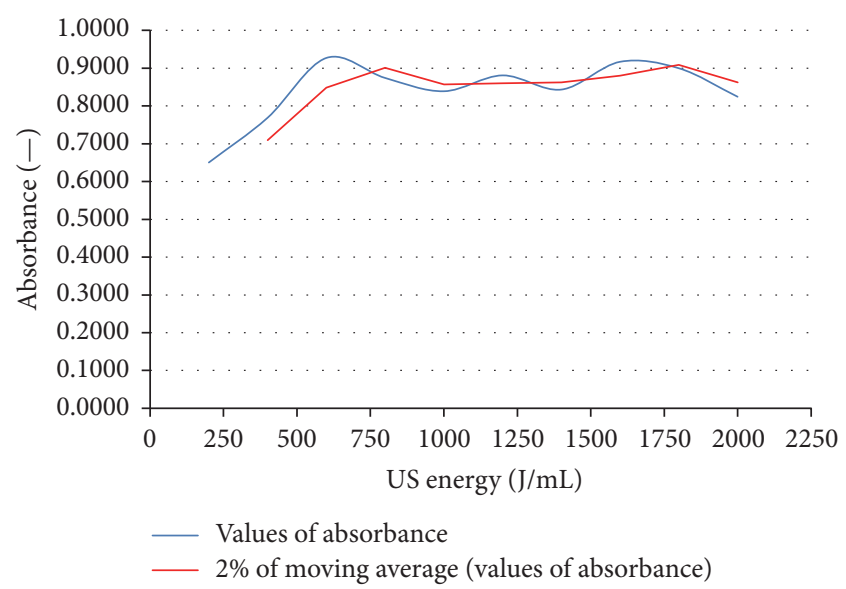

FIGURE 1: Results of absorbance measurement. The optimal value of US energy was determined by finding the curve's inflection point$800 \mathrm{~J} / \mathrm{mL}$. At this point, the absorbance (CNT's dispersion) is at its peak value and further ultrasonication is unnecessary.

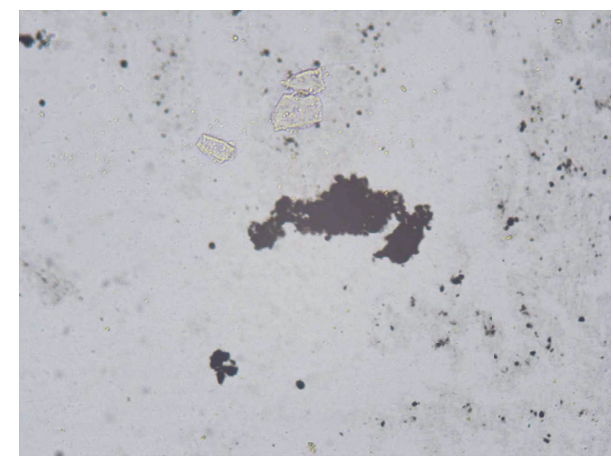

Figure 2: Optical microscope image of CNT dispersion after the treatment with $400 \mathrm{~J} / \mathrm{mL}$ of US energy, magnified 20 times.

be seen in Figures 8(a) and 8(b)). These pictures were taken using Tescan MIRA3 LM scanning electron microscope.

3.3. Results and Discussion. Optimal acoustic energy learned from optical microscopy and UV/Vis spectroscopy observation was determined as $800 \mathrm{~J} / \mathrm{mL}$ as can be seen in Figure 1 . The optimal energy was determined by finding the absorbance curve's inflexion point. The absorbance itself represents the quantity of electromagnetic energy absorbed by dispersion. Generally, the darker the suspension, the higher the absorbance. The final dispersion was once more examined via UV/Vis spectroscopy for its absorbance's verification. The moving average trend curve was chosen as the most accurate in this case.

In Figures 2-5, both effectively dispersed nanotubes and bundled ones can be observed. In Figure $2(400 \mathrm{~J} / \mathrm{mL}$ of US energy), massive bundles are present. In Figure $3(800 \mathrm{~J} / \mathrm{mL}$ of US energy), the well dispersed suspension of nanotubes can be noticed.

As can be seen in Figures 6 and 7, the addition of carbon nanotubes causes an improvement in both flexural and compressive strength in order of percent. In this paper, 


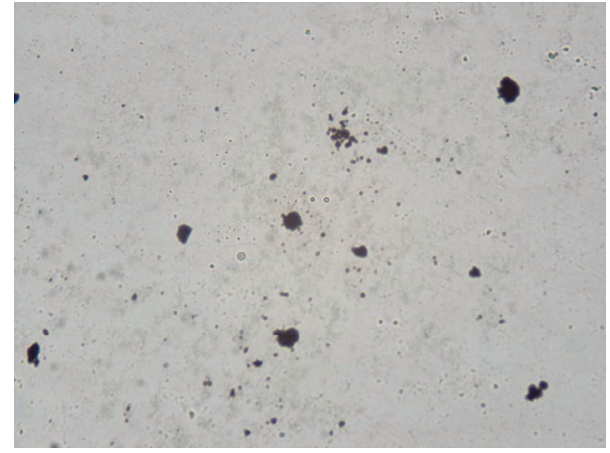

FIGURE 3: Optical microscope image of CNT dispersion after the treatment with $800 \mathrm{~J} / \mathrm{mL}$ of US energy, magnified 20 times.

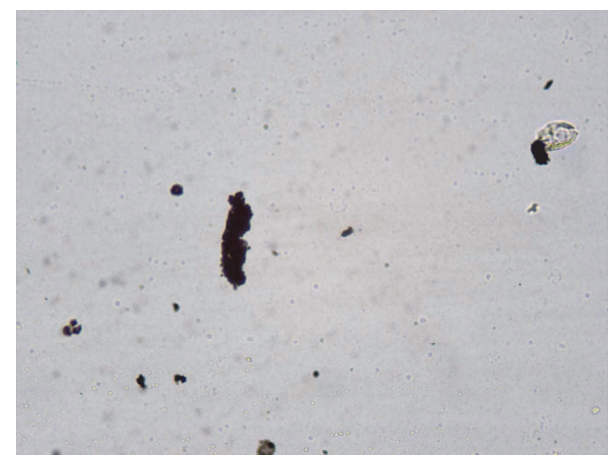

FIgURE 4: Optical microscope image of CNT dispersion after the treatment with $1400 \mathrm{~J} / \mathrm{mL}$ of US energy, magnified 20 times.

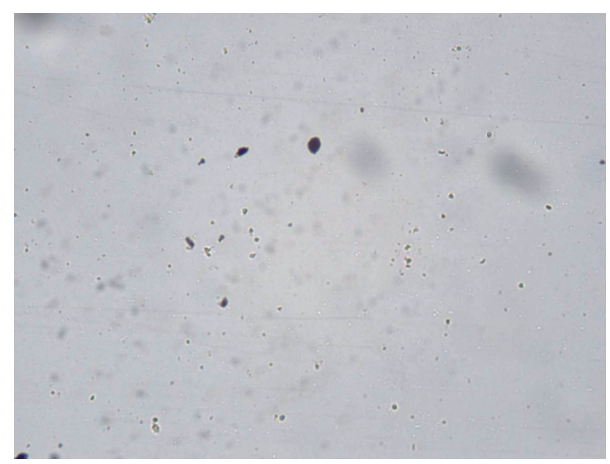

FIgURE 5: Optical microscope image of CNT dispersion after the treatment with $2000 \mathrm{~J} / \mathrm{mL}$ of US energy, magnified 20 times.

relatively low dosage of CNT was used. The reasoning behind this decision was to prove that even with quantity this low, increase in physicomechanical properties could be achieved, if CNT's deagglomeration is sufficient. The results clearly show that even with $0.005 \%$ of CNT (by the weight of cement) it is enough to increase mortar's both flexural and compressive strength. After 7 and 28 days, the $10.4 \%$ and $6.1 \%$ increase in flexural strength was observed, compared to reference specimens. Compressive strength was increased by $5.5 \%$ and $8.6 \%$, respectively.

The reason of strength's (both flexural and compressive) improvement is, according to authors [32-35], the cement

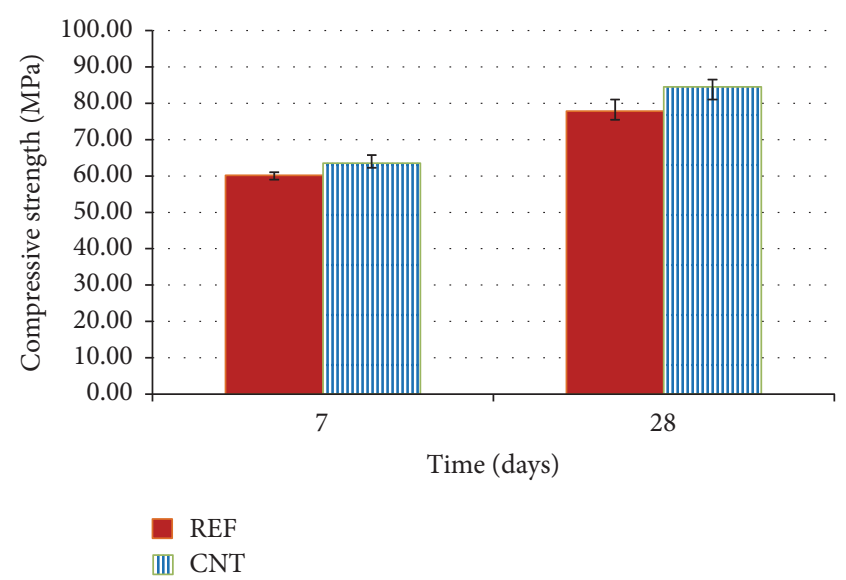

FIGURE 6: Compressive strength of cement mortars with and without CNT after 7 and 28 days. There is obvious increase in mortars' strength with CNT's addition.

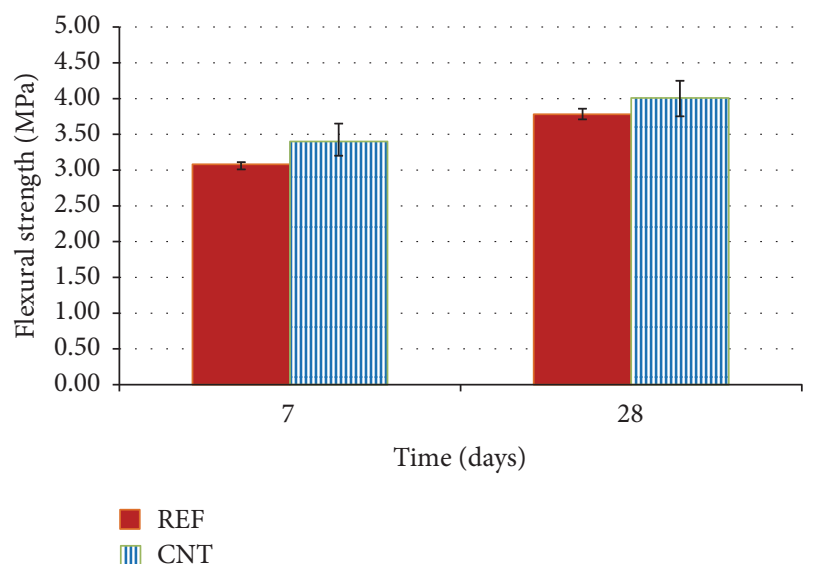

FIGURE 7: Flexural strength of cement mortars with and without CNT after 7 and 28 days. There is obvious increase in mortars' strength with CNT's addition.

matrix's reinforcement with nanotubes. It works on a similar principle, only in macroscale, as reinforcing concrete with steel fibers.

Figures $8(\mathrm{a})$ and $8(\mathrm{~b})$ show the images of cement mortar's microstructure with CNT addition taken by SEM.

\section{Conclusion}

As in many industrial fields, nanotechnology is slowly becoming the construction industry's "next big thing." There are many potentially usable nanoparticles, but probably the most promising ones are carbon nanotubes. These extremely small fibers present both benefits and problems, and one of which was examined in this paper, their dispersion and stability:

(i) With advanced methods like UV/Vis spectroscopy and optical microscopy, the optimal acoustic energy for CNT's effective dispersion was determined, $800 \mathrm{~J} / \mathrm{mL}$. 

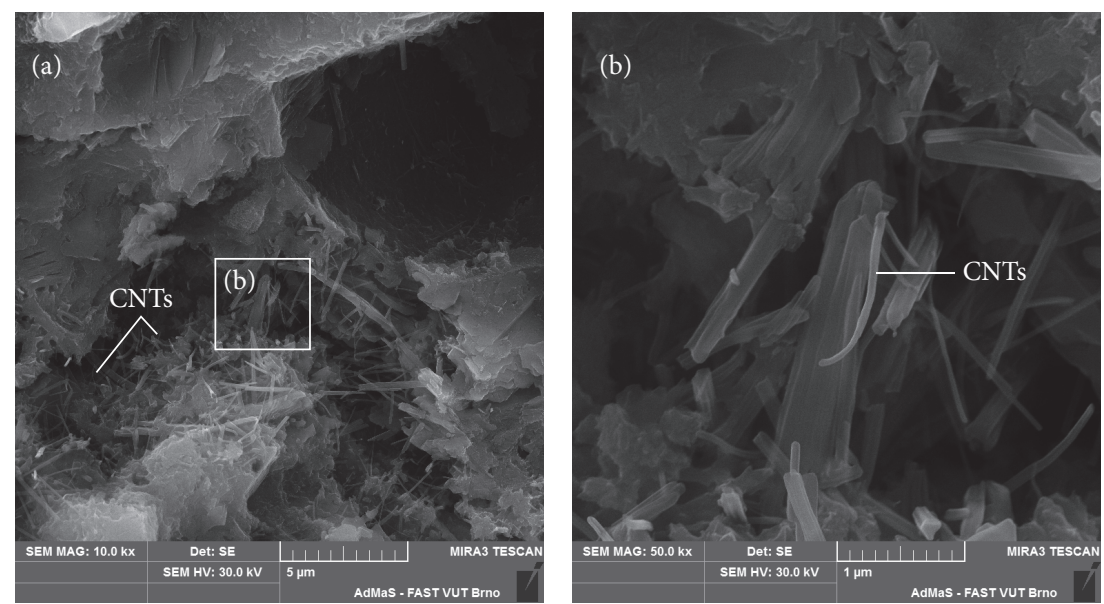

FIGURE 8: (a) SEM images of CNT enhanced cement mortar's microstructure, magnified 10,000 times. (b) SEM images of CNT enhanced cement mortar's microstructure, magnified 50,000 times.

(ii) The CNT mixture was then added to standardized cement mortar and its effect on mechanical properties was tested after 7 and 28 days, respectively.

(iii) The increase in observed physicomechanical properties was from 5.5 to $10.4 \%$.

(iv) An appropriate preparation process of CNT dispersion was specified. The method is, however, not generally applicable. When CNT's parameters (e.g., dimensions, concentration, and purity) change, the whole procedure needs to be repeated [36].

In past decades, research in this area has grown exponentially, and even though we are learning new things every day, there is still plenty of work to do and discoveries to discover.

\section{Competing Interests}

The authors declare that there are no competing interests regarding the publication of this paper.

\section{Acknowledgments}

This paper has been worked out under Project no. LO1408 "AdMaS UP-Advanced Materials, Structures and Technologies," supported by Ministry of Education, Youth and Sports under the "National Sustainability Program I" and under Project GACR P104/15-23219S "Study of methods of nanoparticles dispersion, determination of conditions for preventing their re-agglomeration for application in cement composites" supported by Czech Science Foundation.

\section{References}

[1] R. Hela, J. Marsalova, and L. Bodnarova, "Fly ashes thermal modification and their utilization in concrete," in System-Based Vision For Strategic and Creative Design, F. Bontempi, Ed., vol. 1-3, pp. 1649-1653, 2003.
[2] L. Bodnarova, T. Jarolim, and R. Hela, "Study of effect of various types of cement on properties of cement pastes," Advanced Materials Research, vol. 897, pp. 224-229, 2014.

[3] A. Karaşin and M. Doğruyol, "An experimental study on strength and durability for utilization of fly ash in concrete mix," Advances in Materials Science and Engineering, vol. 2014, Article ID 417514, 6 pages, 2014.

[4] D. Zhang, S. Shi, C. Wang, X. Yang, L. Guo, and S. Xue, "Preparation of cementitious material using smelting slag and tailings and the solidification and leaching of $\mathrm{Pb}^{2+}$, Advances in Materials Science and Engineering, vol. 2015, Article ID 352567, 7 pages, 2015.

[5] K. S. Munir, P. Kingshott, and C. Wen, "Carbon nanotube reinforced titanium metal matrix composites prepared by powder metallurgy-a review," Critical Reviews in Solid State and Materials Sciences, vol. 40, no. 1, pp. 38-55, 2015.

[6] L. Jiang, Z. Li, G. Fan, L. Cao, and D. Zhang, "Strong and ductile carbon nanotube/aluminum bulk nanolaminated composites with two-dimensional alignment of carbon nanotubes," Scripta Materialia, vol. 66, no. 6, pp. 331-334, 2012.

[7] B. Arash, Q. Wang, and V. K. Varadan, "Mechanical properties of carbon nanotube/polymer composites," Scientific Reports, vol. 4, article 6479, 2014.

[8] M. Yoonessi, M. Lebroín-Coloín, D. Scheiman, and M. A. Meador, "Carbon nanotube epoxy nanocomposites: the effects of interfacial modifications on the dynamic mechanical properties of the nanocomposites," ACS Applied Materials \& Interfaces, vol. 6, no. 19, pp. 16621-16630, 2014.

[9] P. A. Danoglidis, M. S. Konsta-Gdoutos, E. E. Gdoutos, and S. P. Shah, "Strength, energy absorption capability and self-sensing properties of multifunctional carbon nanotube reinforced mortars," Construction and Building Materials, vol. 120, pp. 265-274, 2016.

[10] S. Parveen, S. Rana, and R. Fangueiro, "A review on nanomaterial dispersion, microstructure, and mechanical properties of carbon nanotube and nanofiber reinforced cementitious composites," Journal of Nanomaterials, vol. 2013, Article ID 710175, 19 pages, 2013.

[11] M. Bastami, M. Baghbadrani, and F. Aslani, "Performance of nano-Silica modified high strength concrete at elevated 
temperatures," Construction and Building Materials, vol. 68, pp. 402-408, 2014.

[12] A. G. Pérez-Luna, A. L. Martínez-Hernández, G. MartínezBarrera, and C. Velasco-Santos, "Nanoreinforced concrete: effect of gamma-irradiated $\mathrm{SiO}_{2}$ nanoparticles," Advanced Materials Letters, vol. 7, no. 2, pp. 156-162, 2016.

[13] P. Bartos, Nanotechnology of Concrete, Recent Developments and Future Perspectives: Nanotechnology in Construction: A Roadmap for Development, SP-254, American Concrete Institute, Farmington Hills, Mich, USA, 1st edition, 2008.

[14] F. Sanchez and K. Sobolev, "Nanotechnology in concrete-a review," Construction and Building Materials, vol. 24, no. 11, pp. 2060-2071, 2010.

[15] S. Iijima, "Helical microtubules of graphitic carbon," Nature, vol. 354, no. 6348, pp. 56-58, 1991.

[16] N. M. Mubaraka, E. C. Abdullahc, N. S. Jayakumara, and J. N. Sahua, "An overview on methods for the production of carbon nanotubes," Journal of Industrial and Engineering Chemistry, vol. 20, no. 4, pp. 1186-1197, 2014.

[17] J. Yu, N. Grossiord, C. E. Koning, and J. Loos, "Controlling the dispersion of multi-wall carbon nanotubes in aqueous surfactant solution," Carbon, vol. 45, no. 3, pp. 618-623, 2007.

[18] E. N. Ganesh, "Single walled and multi walled carbon nanotube structure, synthesis and applications," International Journal of Innovative Technology and Exploring Engineering, vol. 2, no. 4, pp. 311-320, 2013.

[19] M. Labaj, Suppervisor: R. Hela [Ph.D. thesis], Brno University of Technology, Faculty of Civil Engineering, 2014.

[20] J. Hilding, E. A. Grulke, Z. G. Zhang, and F. Lockwood, "Dispersion of carbon nanotubes in liquids," Journal of Dispersion Science and Technology, vol. 24, no. 1, pp. 1-41, 2003.

[21] J. B. Bai and A. Allaoui, "Effect of the length and the aggregate size of MWNTs on the improvement efficiency of the mechanical and electrical properties of nanocompositesexperimental investigation," Composites Part A: Applied Science and Manufacturing, vol. 34, no. 8, pp. 689-694, 2003.

[22] S. Azoubel and S. Magdassi, "The formation of carbon nanotube dispersions by high pressure homogenization and their rapid characterization by analytical centrifuge," Carbon, vol. 48, no. 12, pp. 3346-3352, 2010.

[23] F. Collins, J. Lambert, and W. H. Duan, "The influences of admixtures on the dispersion, workability, and strength of carbon nanotube-OPC paste mixtures," Cement and Concrete Composites, vol. 34, no. 2, pp. 201-207, 2012.

[24] Technical certificate of normalized sand CEN. According to EN 196-1 Methods of testing cement-part 1: determination of strength, European Committee for Standardization, 2005.

[25] O. Mendoza, G. Sierra, and J. I. Tobón, "Influence of super plasticizer and $\mathrm{Ca}(\mathrm{OH})_{2}$ on the stability of functionalized multiwalled carbon nanotubes dispersions for cement composites applications," Construction and Building Materials, vol. 47, pp. 771-778, 2013.

[26] S. Chuah, Z. Pan, J. G. Sanjayan, C. M. Wang, and W. H. Duan, "Nano reinforced cement and concrete composites and new perspective from graphene oxide," Construction and Building Materials, vol. 73, pp. 113-124, 2014.

[27] T. Jarolím, R. Hela, and M. Labaj, "Influence of the amount of dispersed suspension of carbon nano-tubes on physicomechanical properties of cement mortar," Advanced Materials Research, vol. 1106, pp. 65-68, 2015.
[28] H. Förster, "UV/VIS spectroscopy," in Characterization I, H. G. Karge and J. Weitkamp, Eds., vol. 4 of Molecular Sieves-Science and Technology, pp. 337-426, Springer, Berlin, Germany, 2004.

[29] "Technical certificate of CEM I 42.5 R, Mokra (Heidelberg, Cement Czech Republic)," http://www.heidelbergcement.cz/cs/ cement/volne-lozeny-cement/cemi425r.

[30] EN 196-1 Methods of testing cement-Part 1: Determination of strength, European Committee for Standardization, 2005.

[31] L. Gao, H. Yin, H. Zhu, X. Mao, F. Gan, and D. Wang, "Separation of dispersed carbon nanotubes from water: effect of $\mathrm{pH}$ and surfactants on the aggregation at oil/water interface," Separation and Purification Technology, vol. 129, pp. 113-120, 2014.

[32] T. Nochaiya and A. Chaipanich, "Behavior of multi-walled carbon nanotubes on the porosity and microstructure of cementbased materials," Applied Surface Science, vol. 257, no. 6, pp. 1941-1945, 2011.

[33] S. Xu, J. Liu, and Q. Li, "Mechanical properties and microstructure of multi-walled carbon nanotube-reinforced cement paste," Construction and Building Materials, vol. 76, pp. 16-23, 2015.

[34] J. M. Makar, J. C. Margeson, and J. Luh, "Carbon nanotube/cement composites-early reasults and potential applications," in Proceedings of the 3rd International Conference of Construction Materials, pp. 1-10, Vancouver, Canada, August 2005.

[35] J. M. Makar and G. W. Chan, "Growth of cement hydration products on single-walled carbon nanotubes," Journal of the American Ceramic Society, vol. 92, no. 6, pp. 1303-1310, 2009.

[36] M. S. Konsta-Gdoutosa, Z. S. Metaxaa, and S. P. Shahb, "Highly dispersed carbon nanotube reinforced cement based materials," Cement and Concrete Research, vol. 40, no. 7, pp. 1052-1059, 2010 . 

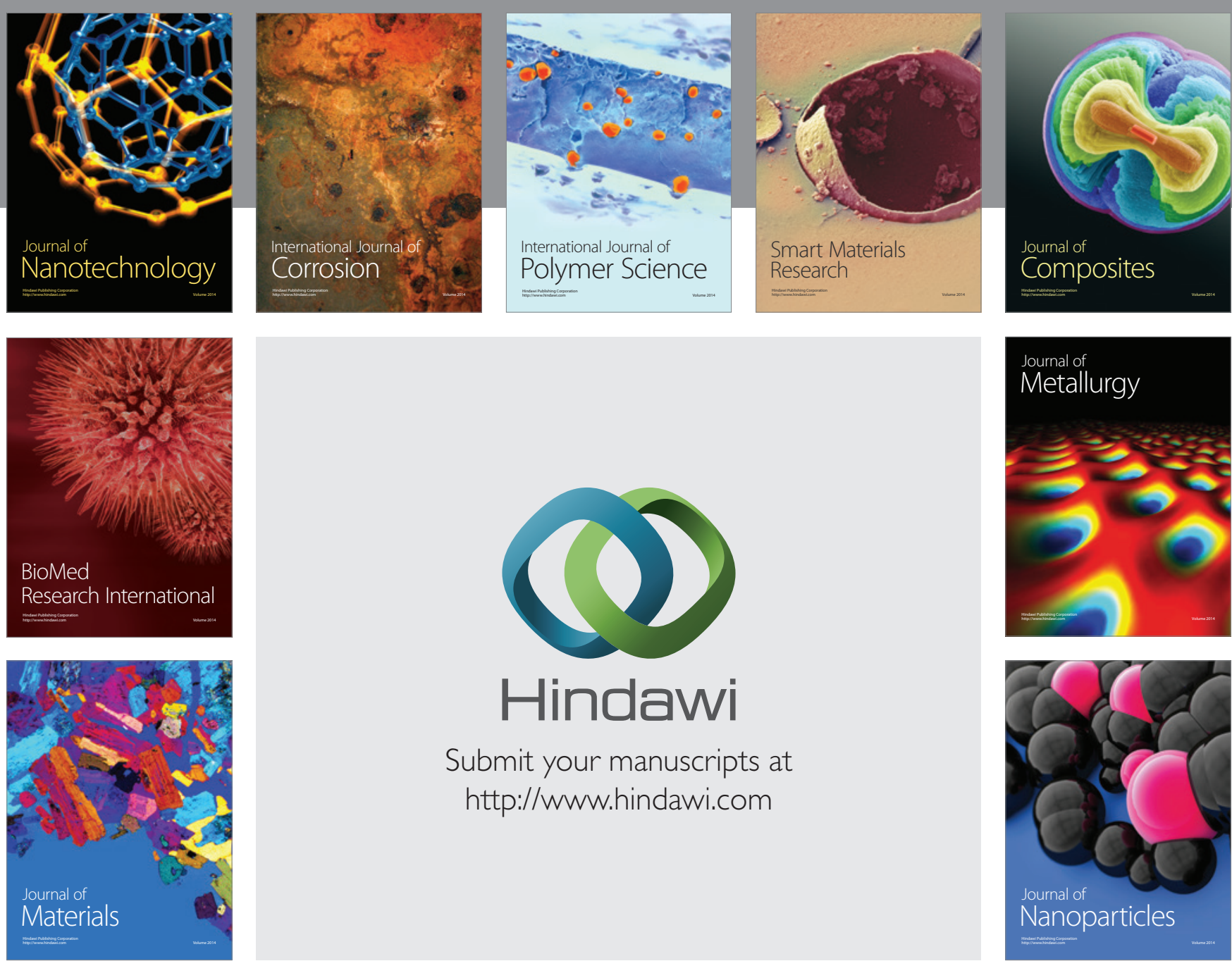

\section{Hindawi}

Submit your manuscripts at

http://www.hindawi.com

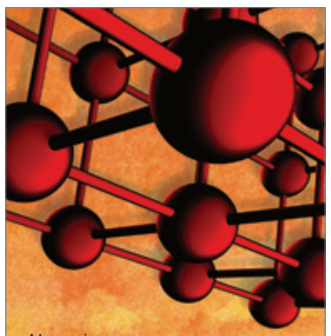

Materials Science and Engineering
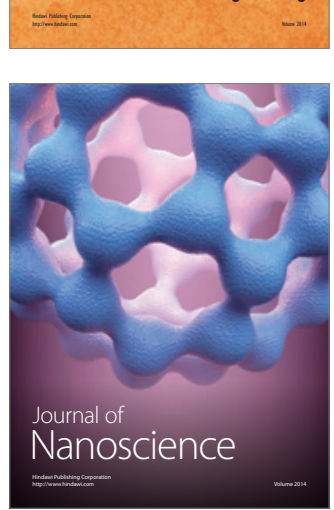
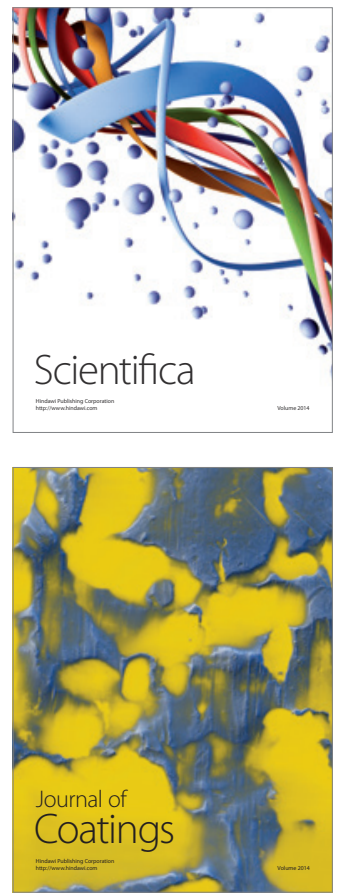
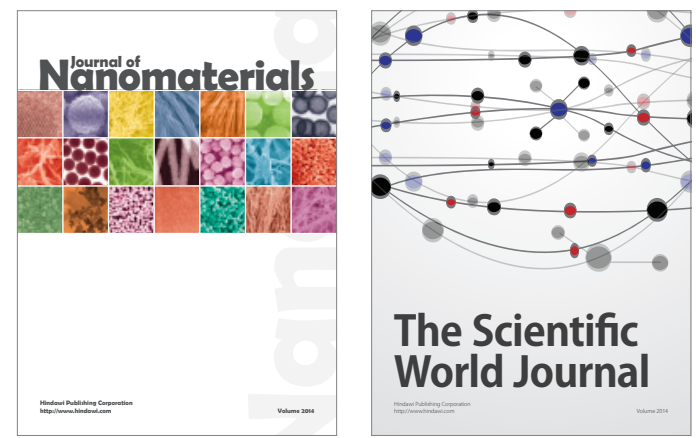

The Scientific World Journal
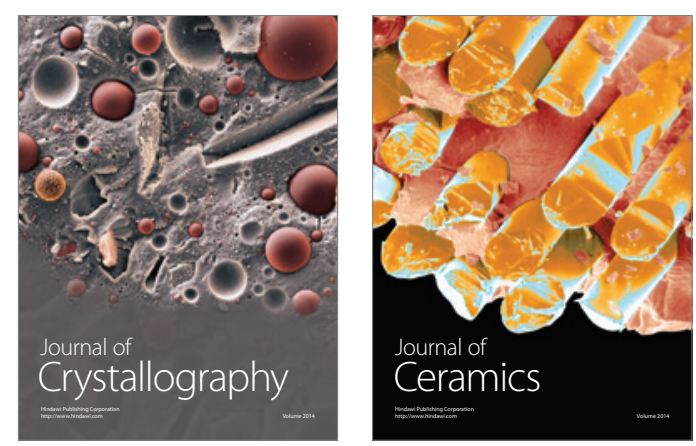
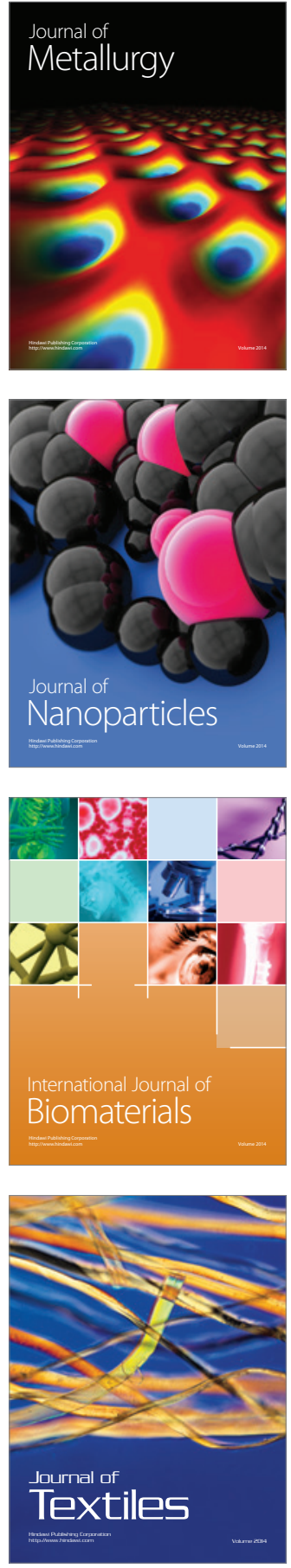